\title{
ORIGINAL
}

\section{The role of colour Doppler ultrasonography in the preoperative localization of parathyroid adenomas}

\author{
Afshin Mohammadi ${ }^{1)}$, Farzad Moloudi ${ }^{2)}$ and Mohammad Ghasemi-rad ${ }^{3)}$ \\ 1) Department of Radiology, Urmia University of Medical Sciences, West-Azerbaijan, Iran \\ 2) Student research committee, Urmia University of medical Sciences, Urmia, Iran \\ 3) Genius and talented student organization, student research committee, Urmia University of medical Sciences, Urmia, Iran
}

\begin{abstract}
The aim of the present study was to evaluate the diagnostic accuracy and imaging patterns of colour Doppler ultrasonography (US) and compare it with grayscale US, 99m-Tc methoxyisobutylisonitrile (MIBI) scans, and combined US and MIBI scans in the preoperative diagnosis of parathyroid adenomas in patients with primary hyperparathyroidism (pHPT). From June 2007 to June 2011, 36 consecutive patients (seven men and 29 women) with pHPT underwent grayscale US, colour Doppler ultrasonography (CDUS), and 99m-Tc MIBI scans prior to parathyroidectomy with traditional unilateral neck dissection. All 36 patients with pHPT underwent parathyroidectomy at our university hospital. According to histopathology results, the sensitivity, specificity, and accuracy of MIBI and US scan were $88 \%, 94 \%$, and $91 \%$, and $70 \%$, $100 \%$, and $85 \%$, respectively. The overall sensitivity and specificity of combined US and MIBI was $97 \%$ and $100 \%$ respectively. The overall sensitivity, specificity, and accuracy of CDUS in the correct diagnosis of parathyroid adenoma were $97 \%, 100 \%$, and $98.6 \%$, respectively. The sensitivity and specificity of US in the detection of parathyroid adenoma and differentiating it from other cervical masses reached up to $97 \%$ and $100 \%$, respectively, by combining CDUS with grayscale evaluations of parathyroid adenoma.
\end{abstract}

Key words: Ultrasonography, Parathyroid, Adenoma

HYPERPARATHYROIDISM is the leading cause of hypercalcemic states and is due to overproduction of the parathyroid hormone by the parathyroid glands (PTGs) [1]. Primary hyperparathyroidism (pHPT) is the third most common endocrine disease after diabetes mellitus and thyroid disease [1, 2]. pHPT is more common in postmenopausal women when compared to the general population $(0.3 \%$ in the general population and $1 \%-3 \%$ in postmenopausal women) $[2,3]$. Over $80 \%$ of patients with pHPT have a single parathyroid adenoma, and the remaining $10-20 \%$ have multiple adenomas, parathyroid hyperplasia, and, rarely, parathyroid carcinoma [1]. Surgical resection of parathyroid adenoma is the only standard treatment for patients with pHPT [3]. Focused unilateral neck exploration - i.e., minimally invasive parathyroidectomy (MIP) - and other minimally invasive

Submitted Nov. 22, 2011; Accepted Jan. 24, 2012 as EJ11-0351 Released online in J-STAGE as advance publication Feb.10, 2012

Correspondence to: Afshin Mohammadi, M.D., Department of Radiology, Urmia University of Medical Sciences, Urmia, WestAzerbaijan, Iran. E-mail: Mohamadi_afshin@yahoo.com approaches, including video-assisted parathyroidectomy and endoscopic parathyroidectomy, are attractive alternatives to total parathyroidectomy $[4,5]$. In all of these focused approaches, the accurate preoperative localization of a parathyroid adenoma is critical prior to performing a surgical resection.

Ultrasonography (US) and 99m-Tc methoxyisobutylisonitrile (MIBI) scans are the routine, standard imaging modalities in the preoperative evaluation of PTG pathology, but both techniques have low specificity in the diagnosis of parathyroid adenomas [6]. The aim of the present study was to evaluate the diagnostic accuracy and imaging patterns of colour Doppler ultrasonography (CDUS) in comparison to grayscale US, MIBI scans, and combined US and MIBI scans in the preoperative diagnosis of parathyroid adenomas in patients with PHPT. The novelty of the present study is that it discusses the new Doppler appearance of parathyroid adenomas rather than just explaining the feeding artery. We also believe that we can help surgeons to more specifically diagnosis parathyroid adenomas when compared to using grayscale US alone. 


\section{Material and Methods}

From June 2007 to June 2011, 36 consecutive patients (seven men and 29 women) with pHPT underwent grayscale US, CDUS, and 99m-Tc MIBI scans prior to parathyroidectomy with traditional unilateral neck dissection.

We prospectively evaluated the imaging data of these patients and compared them to postoperative histopathology results and at least a 50\% reduction in intraoperative parathyroid hormone (PTH) as the gold-standard method for determining the diagnostic accuracy of these imaging modalities in the diagnosis of parathyroid adenoma. The patients' sex, age, and serum intact parathyroid hormone (iPTH) were collected from their records. The university review board approved the study.

We routinely perform CDUS in evaluation of all cervical masses in our department for the determination of the vascularity of cervical masses and their relation to major cervical vessels. We described the vascularity of cervical lesions as having either central, peripheral, combined, uniform, or no vascularity.

A single radiologist with six years' experience performed all of the US examinations with an ultrasound device (Toshiba Nemio 30; Toshiba CO. Ltd, Tokyo, Japan), using a high-frequency (11 MHz) linear array transducer. The patients' hyperextended necks were examined in a supine position from the level of the mandibular angle to the sternal notch. All of the US examinations were performed before the results of the 99m-Tc MIBI scan.

In grayscale sonography, enlarged parathyroid glands appear as round or oval-shaped homogenous hypo-echoic nodules in the posterior part of the thyroid lobes, and are clearly separated from the thyroid lobes by an echogenic ring composed of capsules of adenoma and surrounding fatty tissue (Fig. 1). The nodules with this sonographic appearance were considered to be parathyroid adenomas.

The parathyroid gland size and volume were measured and the location of parathyroid adenomas defined according to US findings. Images were obtained in both transverse and sagittal views. US results were defined as true or false positive and negative according to surgical pathology results. Postoperative histopathology was considered the gold standard for the study.

CDUS and power Doppler ultrasonography (PDUS) were performed by setting the Doppler parameter to detect the vascularity of parathyroid adenoma. In CDUS and PDUS, we evaluate the vascularity of parathyroid gland lesions using the knowledge of an adenoma's blood supply from a separate branch of the inferior thyroid artery.

Colour Doppler findings of each parathyroid lesion were defined as positive or negative on the basis of detection of hypervascularity in the parathyroid gland lesion, subjectively. We classified the lesions at the sites of PTGs according to CDUS and PDUS findings as having no detectable flow (Fig. 2), central hypervascularity (Fig. 3), peripheral hypervascularity (or a ring) (Fig. 4), uniform hypervascularity (what we called a "spot of fire") (Fig. 5), or combined central or peripheral hypervascularity (Fig. 6). The nodules in the parathyroid locations with all of these CDUS or PDUS appearances in the subjective evaluation of parathyroid lesions were considered to be true positive scans. For an objective evaluation we measured the Resistive index of the arterial supply to the adenoma by spectral Doppler sonography (Fig. 7)

In 99m-Tc MIBI scintigraphy, the thyroid images were acquired from an antero-posterior view of the neck and upper part of the thorax over five minutes, using a gamma camera 20 minutes after the intravenous administration of $99 \mathrm{~m}$-Tc pertechnetate $(20 \mathrm{mCi})$. The images were obtained with patients in a supine position.

The parathyroid scan was acquired 15 minutes (thyroid phase) and 120 minutes (parathyroid phase) after intravenous administration of $15-20 \mathrm{mCi}$ of $99 \mathrm{~m}-\mathrm{Tc}$

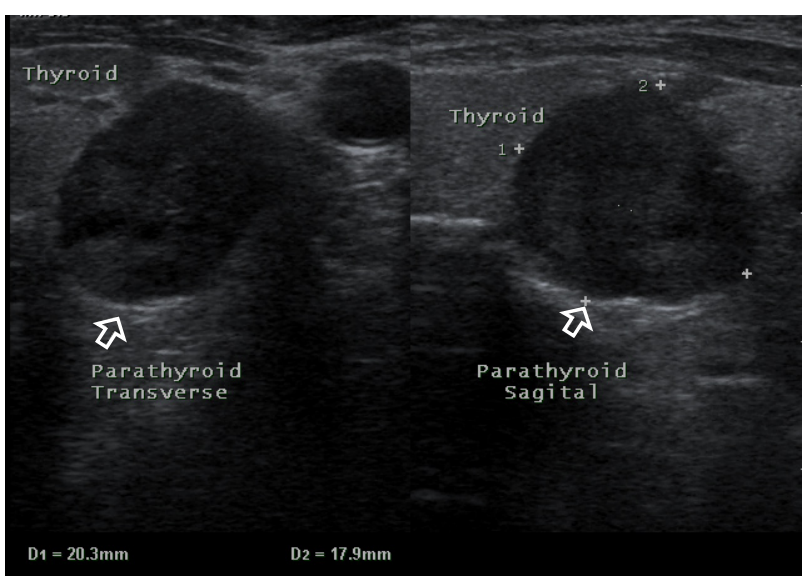

Fig. 1 Ultrasound image showing typical hypoechoic adenoma (arrows) deep in relation to the lower pole of the thyroid in transverse sonogram (A: sagittal and B: transverse views). 


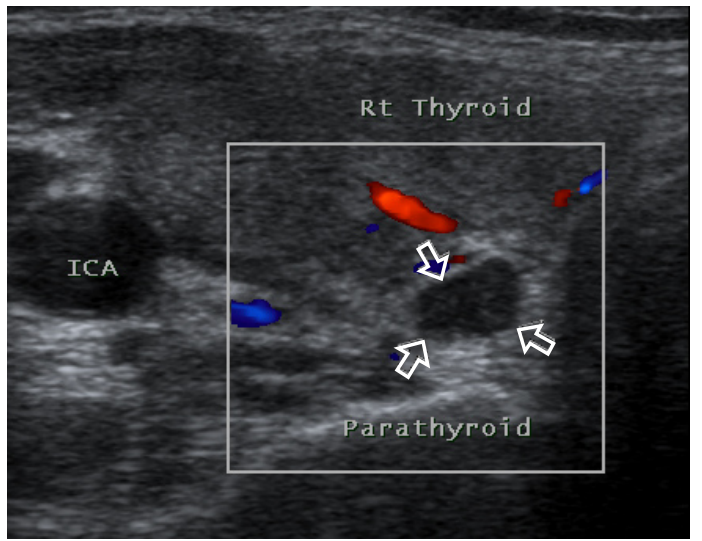

Fig. 2 Colour Doppler sonogram shows typical hypoechoic adenoma deep in relation to the lower pole of the thyroid without a detectable flow in a colour Doppler sonogram.

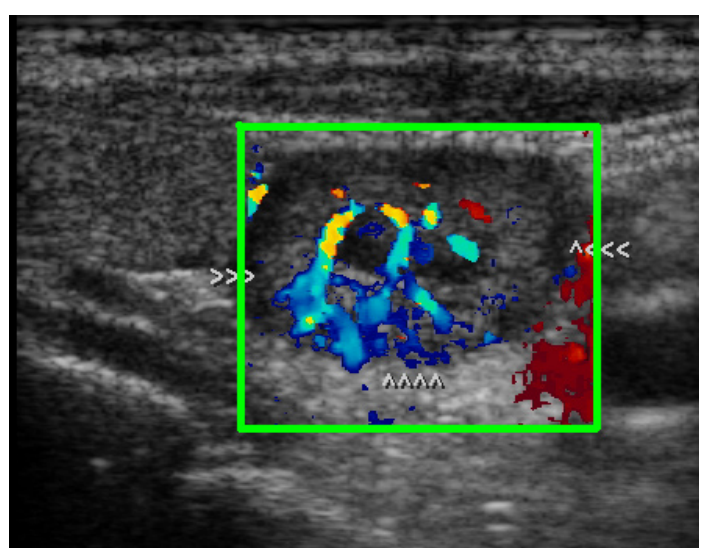

Fig. 3 Colour Doppler sonogram shows a typical hypoechoic adenoma deep in relation to the lower pole of the thyroid with central vascularity in a colour Doppler sonogram.

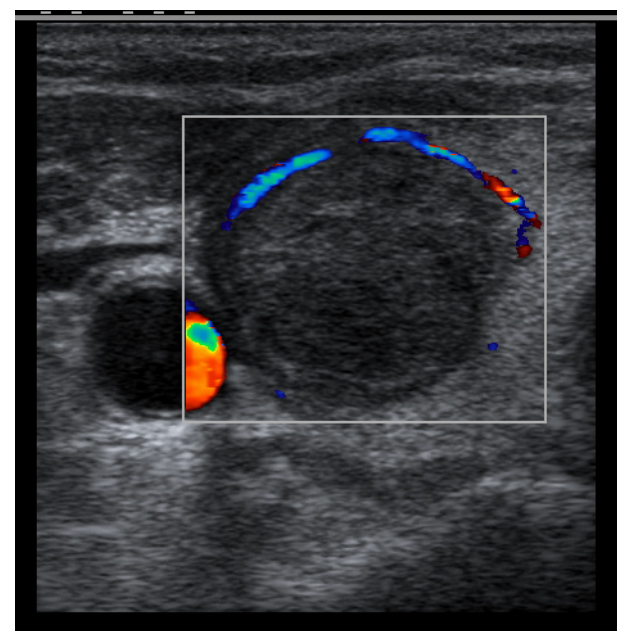

Fig. 4 Colour Doppler sonogram shows a typical hypoechoic adenoma deep in relation to the lower pole of the thyroid, with ring-pattern vascularity in a colour Doppler sonogram.

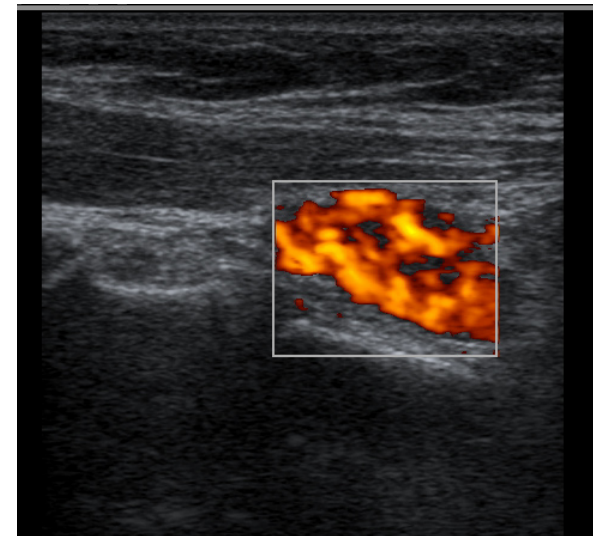

Fig. 5 Colour Doppler sonogram shows a typical spot of fire pattern vascularity in a power Doppler sonogram.

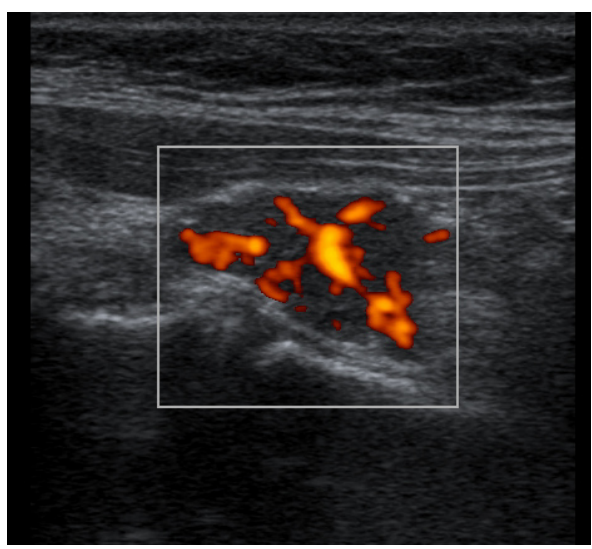

Fig. 6 Doppler sonogram shows a round hypoechoic intrathyroidal adenoma, deep with a combined central and peripheral vascularity in a colour Doppler sonogram.

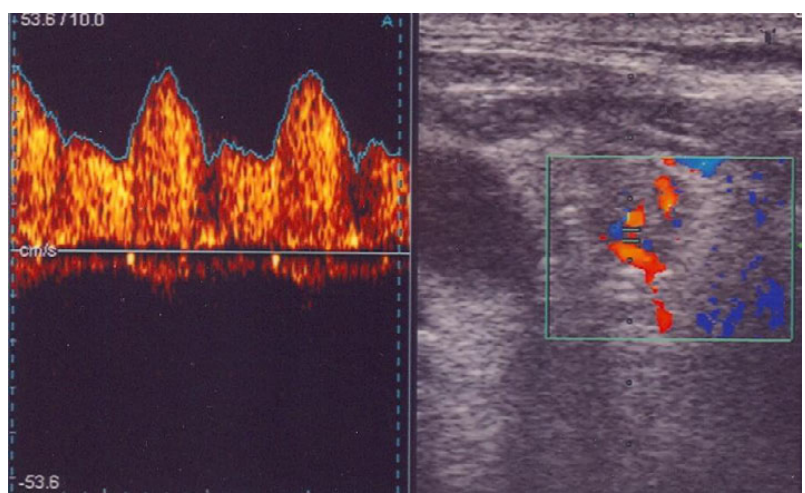

Fig. 7 Spectral Doppler sonogram revealed the low resistance blood flow to the parathyroid adenoma $(\mathrm{RI}=0.56)$ 
MIBI. The images were obtained with a single-head gamma camera (ADAK, Epic, Netherlands) over 10 minutes, using a pinhole collimator. The MIBI scan was considered to be positive for parathyroid lesions when an increase in uptake was detected in the thyroid phase.

The 99m-Tc MIBI single-photon emission computed tomography (SPECT) was performed 20-30 minutes after IV injection, and reconstructed to produce threedimensional projections. The SPECT was performed in a full anterior (180-degree) view as 32 frames, at 30 seconds per frame. Focal increased uptake in the parathyroid site was considered positive. Our nuclear physician specialist was blinded to the results of ultrasound examination.

Statistical analysis was performed using SPSS statistical analysis software (version 16, Chicago, IL, USA). Continuous variables were reported as means $\pm \mathrm{SD}$, and categorical variables as frequencies. Since the distribution of PTH and volume was not normal, a nonparametric spearman's test was used for analysis. One-way ANOVA, a chi-square test, and a multivariate linear model were used for the evaluation of variables.

\section{Results}

All 36 patients (female-to-male ratio of 4:1) with pHPT underwent parathyroidectomy at our university hospital. The mean age was $52.2 \pm 12.6$ years.

Skeletal involvement, especially bone pain, was the most common presenting symptom of our patients (70\%). The bone pain and limb claudication due to a giant brown tumour in the iliac wing were the first presenting symptoms in two patients. Renal calculi, including nephrocalcinosis, were present in $(44 \%)$ patients. Proximal muscle weakness was seen in $48 \%$ of our patients. Parathyroid adenoma was palpable on careful examination in three patients. None of our patients had psychiatric symptoms or obvious neurological problems. Gastrointestinal symptoms such as nausea, vomiting, and constipation were seen in three patients, and intractable vomiting was the major presenting sign in one male patient.

The mean preoperative serum calcium was elevated $(12.64 \pm 1.33 \mathrm{mg} / \mathrm{dL}$, ranging from $10.7-17 \mathrm{mg} /$ $\mathrm{dL}$ ), and the patients had low inorganic phosphorus levels $(2.11 \pm 0.56 \mathrm{mg} / \mathrm{dL})$. The mean postoperative serum calcium was $8.9 \pm 1.02 \mathrm{mg} / \mathrm{dL}$. The mean preoperative serum iPTH level was $584 \pm 510 \mathrm{pg} / \mathrm{mL}$

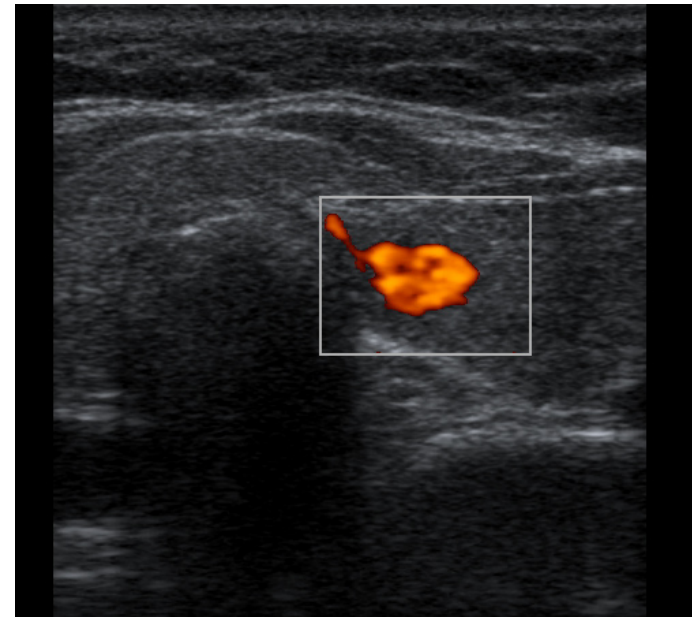

Fig. 8 Colour Doppler sonogram shows a hypervascular intrathyroidal adenoma with a spot-of-fire appearance.

(ranging from $80-2000 \mathrm{pg} / \mathrm{mL}$ ), and the mean postoperative serum iPTH level was $52 \pm 5.3 \mathrm{pg} / \mathrm{mL}$ (ranging from 39-68 $\mathrm{pg} / \mathrm{mL}$ ). The mean intraoperative serum iPTH was $129 \pm 61 \mathrm{pg} / \mathrm{mL}$ that revealed $78 \%$ decrease in serum iPTH 20 minutes after adenoma excision. The mean volume of parathyroid adenomas was $2.18 \pm 2.03$ cc (ranging from 0.2-7.5 cc).

Three patients with pHPT had intra-thyroidal parathyroid adenomas; all were identified via MIBI scans. US were negative in all three patients due concomitant multinodular goitres. All three intrathyroidal nodules had significant hypervascularity in CDUS (Fig. 8). Histopathology revealed that one US-confirmed parathyroid lesion was a lymph node that had not hypervascularity in CDUS, and one lesion was an exophytic thyroid nodule mimicking a parathyroid adenoma on grayscale sonography but it had hypervascularity in CDUS compatible with parathyroid adenoma.

According to CDUS and PDUS, 35 adenomas were detected correctly. Seventeen had ring vascularity with a mean volume of $2.12 \pm 1.88 \mathrm{cc}$, two had central vascularity with a mean volume of $2.2 \pm 1.83 \mathrm{cc}$, and nine had a combined pattern with a mean volume of $3.84 \pm 2.06 \mathrm{cc}$. The remaining adenomas (7 of 35) had uniform hypervascularity in CDUS and PDUS, what we called a "spot of fire," with a mean volume of $0.47 \pm 0.3 \mathrm{cc}$. Only one parathyroid adenoma, with a volume of $0.2 \mathrm{cc}$, had no flow on CDUS.

According to histopathology results, Table 1 summarizes the diagnostic usefulness of each modality in the diagnosis of parathyroid adenoma. Our study 
Table 1

\begin{tabular}{ccccc}
\hline Modality & US & MIBI & $\begin{array}{c}\text { Combined } \\
\text { US/MIBI }\end{array}$ & CDUS \\
\hline Sensitivity & $88 \%$ & $70 \%$ & $97 \%$ & $97 \%$ \\
Specificity & $94 \%$ & $100 \%$ & $100 \%$ & $100 \%$ \\
Accuracy & $91 \%$ & $85 \%$ & $98.6 \%$ & $98.6 \%$ \\
\hline
\end{tabular}

Table 2

\begin{tabular}{cccc}
\hline Variable & MIBI & MIBI & \multirow{2}{*}{ Posalue } \\
& Positive & Negative & \\
\hline iPTH (pg/mL) & $666 \pm 554$ & $222 \pm 146$ & $P<0.025$ \\
Volume (cc) & $2.42 \pm 2.09$ & $1.6 \pm 1.94 \mathrm{cc}$ & $P=0.3$ \\
\hline
\end{tabular}

showed that there is a significant difference between serum iPTH levels and positive MIBI scans, but the mean volume of parathyroid lesions did not show significant differences between MIBI-positive and MIBInegative patients. Table 2 summarizes the relationship between MIBI and parathyroid adenoma volumes and serum iPTH levels.

We found that there is a positive correlation between the volume of parathyroid adenomas and serum iPTH levels ( $\mathrm{r}=0.35, P<0.035)$. The mean RI of parathyroid adenoma blood flows was $0.55 \pm 0.06$, which revealed a low-resistance blood flow.

\section{Discussion}

Most of the healthy adult members of the population have four PTGs, less than 5\% have fewer than four, and $3 \%$ have more than four $[7,8]$. The average PTG dimension is $5 \times 3 \times 1 \mathrm{~mm}$, and the average weight is $30-40 \mathrm{mg}[7,8]$.

Recently, we encountered an increase in the prevalence of pHPT. The prevalence of pHPT reached $0.67 \%$ in 2006 , compared to a previous report of $0.18 \%$ in 1997. It is more-frequently diagnosed in females than in males, the incidence increases with age, and mostly occurs between the ages of 40 and $60[9,10]$. Yu et al. [11] showed that patients with pHPT have an increased risk of all-cause mortality and cardiovascular mortality even with mild untreated PHPT.

Currently, focused unilateral cervical parathyroidectomy and other MIPs, such as video-assisted parathyroidectomy and endoscopic parathyroidectomy, have made the accurate preoperative localization of parathyroid disease essential to a satisfactory surgical outcome [4]. Today, US and 99mTc-sestamibi scintigraphy are the routine modalities for preoperative localization of parathyroid adenomas, especially when both techniques are used together preoperatively $[12,13]$. However, in ectopic parathyroid adenomas in mediastinal locations, hybrid techniques like single-photon emission computed tomography/computed tomography are the most accurate approaches to use [14].

Hyperparathyroidism is commonly accompanied by thyroid disease, and reactive lymph nodes may be particularly prominent in these patients. Therefore, reactive lymph nodes can be mistaken for parathyroid adenomas or hyperplasia [8].

In our study, the prevalence of parathyroid adenomas was more frequent in females than in males. The mean age of our patients was $52.2 \pm 12.6$ years, which agrees with previous data [16, 20].

According to our last search, the mean parathyroid adenoma gland size and serum iPTH level in pHPT cases in our study agreed with the study in Europe, the United States, Asia, and South America [19, 21-24]. Despite the mean gland size, there were eight parathyroid adenomas with a volume under 0.6 cc in our study that was still correctly diagnosed by colour Doppler sonography. Although our study is mainly subjective, it showed that parathyroid adenomas have a very lowresistance blood flow, which can be an objective confirming measurements in the diagnosis of parathyroid adenomas. In our study, the diagnostic accuracy of a combination of US and MIBI scans was higher than each alone, making our results in agreement with previous studies [25-27].

There was a significant difference in serum iPTH levels in patients with MIBI-positive results compared to the MIBI-negative group ( $p=0.039$ ). Previous articles showed many controversies about the relationship between serum iPTH levels and MIBI scans. Some previous investigations have demonstrated a relationship between serum iPTH levels and MIBI results [28-30], while others have not shown such a correlation [31]. Sukan et al. [19] showed that there was a significant difference in intact PTH levels between MIBI-positive and -negative patients.

We found a correlation between serum iPTH levels and ultrasonographic estimations of parathyroid adenoma volumes. Our results contrast those of another study, which did not show a correlation between serum iPTH and the weight of the parathyroid glands [19, 20]; however, they are in agreement with another 
study that did show a correlation between serum iPTH and the weight of parathyroid glands [28]. Kakuta et al. showed that the volume of PTGs given by US is a good indicator of their weight: larger PTGs secrete more whole iPTH per gland [28].

We did not find a relationship between ultrasonographic PTG volumes and MIBI results $(p=0.3)$. This agrees with the findings of a study by Kasai et al., which reported that a negative correlation between gland weight and MIBI uptake were not significant [29]. They reported that the overall sensitivity of MIBI and US scans was $84 \%$ and $72.5 \%$, respectively [29].

A study by Kebapci et al. showed that, in patients without concomitant thyroid disease, US alone should be used as a first step for preoperative localization, and the combination of US and MIBI scintigraphy is a reliable localization technique in patients with concomitant thyroid disease [30]. Wolf et al. reported that 63\% of parathyroid adenomas had a vascular arc in CDUS (90 to 270 degrees), and that the identification of a vascular arc improves the diagnostic specificity of parathyroid adenomas [31].

Rickes et al. [32] and Lane et al. [33] reported that the detection of feeding vessels by CDUS is an important indication of a parathyroid lesion, and that these vessels can serve as "road maps" to abnormal parathyroid glands. We found that, although in some cases feeding may not be detectable, there are some other patterns that could be helpful.

Kamaya et al. [34] found that an enlarged feeding artery and peripheral arc of vascularity seen on CDUS and PDUS are the typical imaging characteristics of parathyroid adenoma in sonographic evaluation. Reeder et al. [35] found that a combination of CDUS and PDUS with grayscale imaging was required for the sonographic localization and identification of small parathyroid adenomas.

If a parathyroid adenoma is not located in the upper or lower gland locations (ectopic), the correct preoperative localization of parathyroid adenomas with routine imaging modalities will be challenging. It was recently revealed that the single-photon emission computed tomography/computed tomography (SPECT/ CT) fusion technique offers the advantage of combining function and anatomy for the exact localization of ectopic parathyroid adenomas. SPECT/CT permitted the highest reader confidence in the localization of the carotid sheath, anterior mediastinum, retropharynx, or intrathyroidal ectopic parathyroid adenomas [36].

Another diagnostic difficulty in the preoperative localization of parathyroid adenomas is concomitant thyroid nodules; a previous study [30] showed that, in patients without thyroid disease, the sensitivity of these techniques was $90 \%$ and $75 \%$, respectively. In patients with thyroid disease, the sensitivity was $78 \%$ and $70 \%$, respectively. Localization of typical blood supply patterns on colour Doppler imaging and the diagnosis of feeding arteries derived from the inferior thyroidal artery can be helpful in differentiating PTGs from thyroid nodules.

There is two major differential diagnosis for parathyroid adenoma that may be mistake in grayscale sonograghy, lymph node and thyroid nodule. Ying et al. showed that only high resistive central vascularity was found in cervical nodes that are more prominent in submental region [37]. Shimamoto et al. showed the perinodular flow in 10 patients from 13 patients [38]. According to our experience revealed that prominent hypervascularity in comparison with other cervical structure can aid in differentiation of parathyroid adenoma from lymph nodes and thyroid nodules.

\section{Conclusion}

Parathyroid adenomas demonstrate different imaging patterns in CDUS. These patterns include central, ring (most common), combined, and uniform (spots of fire) patterns; they are highly specific for parathyroid adenoma and can differentiate it from other cervical masses. Parathyroid adenoma is usually more vascular than other cervical soft tissue components.

Our study revealed that the sensitivity and specificity of US in the detection and differentiation of parathyroid adenoma from other cervical masses reaches up to $97 \%$ and $100 \%$ by combining CDUS with the grayscale sonographic evaluation of parathyroid adenoma. Since US is cheap, non-invasive, and easy to perform, we recommend using US as a first modality, and suggest that it should always be combined with CDUS to increase the diagnostic value; the use of US should be followed by a MIBI scan in case it is not diagnostic. 


\section{References}

1. MacKenzie-Feder J, Sirrs S, Anderson D, Sharif J, Khan A (2011) Primary Hyperparathyroidism: An Overview. Int J Endocrinol 251410, Epub 2011.

2. Mihai R, Wass JA, Sadler GP (2008) Asymptomatic hyperparathyroidism - need for multicentre studies. Clin Endocrinol (Oxf) 68: 155-164.

3. Wermers RA, Khosla S, Atkinson EJ (2006) Incidence of primary hyperparathyroidism in Rochester, Minnesota, 1993-2001: an update on the changing epidemiology of the disease. J Bone Miner Res 21:171-177.

4. Carling T, Udelsman R (2008) Focused approach to parathyroidectomy. World J Surg 32:1512-1517.

5. Starker L, Fonseca A, Carling T, Udelsman R (2011) Minimally Invasive Parathyroidectomy. Int J Endocrinol 206502, Epub 2011.

6. Akbaba G, Berker D, Isik S, Aydin Y, Ciliz D,et al (2011) A comparative study of preoperative imaging methods in patients with primary hyperparathyroidism: US, MIBI, SPECT and MRI. J Endocrinol Invest May 27 (Epub ahead of print).

7. Nguyen Ba D (1999) Parathyroid Imaging with Tc-99m Sestamibi Planar and SPECT Scintigraphy. Radiographics 19:601-614.

8. Johnson NA, Tublin ME, Ogilvie JB (2007) Parathyroid Imaging: Technique and Role in the Preoperative Evaluation of Primary Hyperparathyroidism. AJR Am J Roentgenol 188:1706-1715.

9. Yu N, Donnan PT, Murphy MJ, Leese GP (2009) Epidemiology of primary hyperparathyroidism in Tayside, Scotland, UK. Clin Endocrinol (Oxf) 71:485493.

10. Suh JM, Cronan JJ, Monchik JM (2008) Primary hyperparathyroidism: is there an increased prevalence of renal stone disease? AJR Am J Roentgenol 191:908-911.

11. Yu N, Donnan PT, Flynn RW, Murphy MJ, Smith D, et al (2010) Increased mortality and morbidity in mild primary hyperparathyroid patients. The Parathyroid Epidemiology and Audit Research Study (PEARS). Clin Endocrinol (Oxf) 73:30-34.

12. Ruda JM, Hollenbeak CS, Stack BC Jr (2005) A systematic review of the diagnosis and treatment of primary hyperparathyroidism from 1995 to 2003. Otolaryngol Head Neck Surg 132:359-372.

13. De Feo ML, Colagrande S, Biagini C, Tonarelli A, Bisi G, et al (2000) Parathyroid glands: combination of (99m) Tc MIBI scintigraphy and US for demonstration of parathyroid glands and nodules. Radiology 214:393340.

14. Levine DS, Belzberg AS, Wiseman SM (2009) Hybrid SPECT/CT imaging for primary hyperparathyroidism: case reports and pictorial review. Clin Nucl Med 34:779-784.

15. Hung GU, Wang SJ, Lin WY (2003) Tc-99m MIBI para- thyroid scintigraphy and intact parathyroid hormone levels in hyperparathyroidism. Clin Nucl Med 28:180185.

16. Ambrosoni P, Heuguerot C, Olaizola I, Acuña G, Fajardo L, et al (1998) Can we use 99mTc-MIBI in functional studies of the parathyroid gland? Nephrol Dial Transplant 13 Suppl 3:33-36.

17. Stawicki SP, El Chaar M, Baillie DR, Jaik NP, Estrada FP (2007) Correlations between biochemical testing, pathology findings and preoperative sestamibi scan: a retrospective study of the minimally invasive radioguided parathyroidectomy (MIRP) approach. Nucl Med Rev Cent East Eur 10:82-86.

18. Carpentier A, Jeannotte S, Verreault J, Lefebvre B, Bisson G, et al (1998) Preoperative localization of parathyroid lesions in hyperparathyroidism: relationship between technetium-99m-MIBI uptake and oxyphil cell content. J Nucl Med 39:1441-1444.

19. Sukan A, Reyhan M, Aydin M, Yapar AF, Sert Y, et al (2008) Preoperative evaluation of hyperparathyroidism: the role of dual-phase parathyroid scintigraphy and ultrasound imaging. Ann Nucl Med 22:123-131.

20. Saxe AW, Lincenberg S, Hamburger SW (1987) Can the volume of abnormal parathyroid tissue be predicted by preoperative biochemical measurement? Surgery 102:840-845.

21. Alexandrides TK, Kouloubi K, Vagenakis AG, Yarmenitis S, Spyridonidis T, et al (2006) The value of scintigraphy and ultrasonography in the preoperative localization of parathyroid glands in patients with primary hyperparathyroidism and concomitant thyroid disease. Hormones 5:42-51.

22. Gopal RA, Acharya SV, Bandgar T, Menon PS, Dalvi AN, et al (2010) Clinical profile of primary hyperparathyroidism from western India: A single center experience. J Postgrad Med 56:79-84.

23. De Feo ML, Colagrande S, Biagini C, Tonarelli A, Bisi G, et al (2000) Parathyroid glands: combination of (99m)Tc MIBI scintigraphy and US for demonstration of parathyroid glands and nodules. Radiology 214:393402.

24. Erbil Y, Barbaros U, Tükenmez M, Işsever $H$, Salmaslioğlu A, et al (2008) Impact of adenoma weight and ectopic location of parathyroid adenoma on localization study results. World J Surg 32:566-571.

25. Lumachi F, Zucchetta P, Marzola MC (2000)Advantages of combined technetium-99m-sestamibi scintigraphy and high-resolution ultrasonography in parathyroid localization: comparative study in 91 patients with primary hyperparathyroidism. Eur J Endocrinol 143:755760.

26. Siperstein A, Berber E, Mackey R, Alghoul M, Wagner K (2004) Prospective evaluation of sestamibi scan, 
ultrasonography, and rapid PTH to predict the success of limited exploration for sporadic primary hyperparathyroidism. Surgery 136:872-880.

27. Jones JM, Russell CF, Ferguson WR, Laird JD (2001) Preoperative sestamibi-technetium subtraction scintigraphy in primary hyperparathyroidism: experience with 156 consecutive patients. Clin Radiol 56:556-559.

28. Kakuta T, Tanaka R, Kanai G, Miyamoto Y, Inagaki M, et al (2008) Relationship between the weight of parathyroid glands and their secretion of parathyroid hormone in hemodialysis patients with secondary hyperparathyroidism. Ther Apher Dial 12:385-390.

29. Kasai ET, da Silva JW, Mandarim de Lacerda CA, Boasquevisque E (2008) Parathyroid glands: combination of sestamibi-(99m)Tc scintigraphy and ultrasonography for demonstration of hyperplasic parathyroid glands. Rev Esp Med Nucl 27:8-12.

30. Kebapci M, Entok E, Kebapci N, Adapinar B (2004) Preoperative evaluation of parathyroid lesions in patients with concomitant thyroid disease: role of high-resolution ultrasonography and dual phase technetium 99m sestamibi scintigraphy. J Endocrinol Invest 27:24-30.

31. Wolf RJ, Cronan JJ, Monchik JM (1994) Color Doppler sonography: an adjunctive technique in assessment of parathyroid adenomas. J Ultrasound Med 13:303-308.

32. Rickes S, Sitzy J, Neye H, Ocran KW, Wermke W
(2003) High-resolution ultrasound in combination with colour-Doppler sonography for preoperative localization of parathyroid adenomas in patients with primary hyperparathyroidism. Ultraschall Med 24:85-89.

33. Lane MJ, Desser TS, Weigel RJ, Jeffrey RB Jr (1998) Use of color and power Doppler sonography to identify feeding arteries associated with parathyroid adenomas. AJR Am J Roentgenol 171:819-823.

34. Kamaya A, Quon A, Jeffrey RB (2006) Sonography of the abnormal parathyroid gland. Ultrasound Q 22: 253262.

35. Reeder SB, Desser TS, Weigel RJ, Jeffrey RB (2002) Sonography in primary hyperparathyroidism: review with emphasis on scanning technique. J Ultrasound Med 21:539-542.

36. Akram K, Parker JA, Donohoe K, Kolodny G (2009) Role of single photon emission computed tomography/ computed tomography in localization of ectopic parathyroid adenoma: a pictorial case series and review of the current literature. Clin Nucl Med 34:500-502.

37. Ying M, Ahuja A, Brook F, Metreweli C (2000) Power Doppler sonography of normal cervical lymph nodes. $J$ Ultrasound Med 19:511-517.

38. Shimamoto K, Endo T, Ishigaki T, Sakuma S, Makino N (1993) Thyroid nodules: evaluation with color Doppler ultrasonography. J Ultrasound Med 12:673-678. 